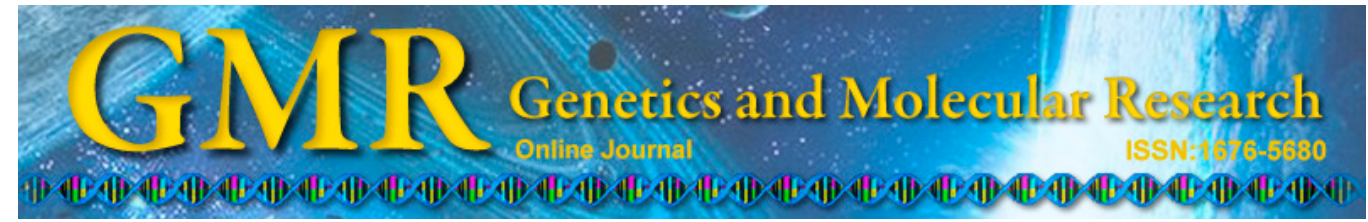

\title{
A rapid and sensitive loop-mediated isothermal amplification procedure (LAMP) for Mycoplasma hyopneumoniae detection based on the $p 36$ gene
}

M.J. Liu ${ }^{1,3}$, G.M. Du ${ }^{1,2}$, F.F. Bai ${ }^{1}$, Y.Z. Wu ${ }^{1}$, Q.Y. Xiong ${ }^{1}$, Z.X. Feng ${ }^{1}$, B. Li ${ }^{1}$ and G.Q. Shao ${ }^{1,4}$

${ }^{1}$ Key Laboratory of Veterinary Biological Engineering and Technology, Institute of Veterinary Medicine, Jiangsu Academy of Agricultural Sciences, Ministry of Agriculture,

National Center for Engineering Research of Veterinary Bio-Products, Nanjing, China

${ }^{2}$ Department of Animal Science and Technology, Jinling Technology Institution, Nanjing, China

${ }^{3}$ Key Laboratory of Animal Physiology and Biochemistry, Ministry of Agriculture, Nanjing Agricultural University, Nanjing, China ${ }^{4}$ Jiangsu Co-Innovation Center for Prevention and Control of Important Animal Infectious Diseases and Zoonoses, Yangzhou, China

Corresponding author: G.Q. Shao

E-mail: gqshaojaas@gmail.com

Genet. Mol. Res. 14 (2): 4677-4686 (2015)

Received September 5, 2014

Accepted November 6, 2014

Published May 4, 2015

DOI http://dx.doi.org/10.4238/2015.May.4.27

ABSTRACT. The aim of this study was to establish a method for sensitive and rapid diagnosis of Mycoplasma hyopneumoniae in clinical specimens. To this effect, we employed three sets of primers specifically designed for amplification of nucleic acids under isothermal conditions. After optimization of reaction conditions, M. hyopneumoniae could be successfully detected at $63^{\circ} \mathrm{C}$ in $45 \mathrm{~min}$ through use of the loop- 
mediated isothermal amplification (LAMP) assay. A positive reaction was identified visually as white precipitate and confirmed by gel electrophoresis. The detection limit for this assay was 10 copies $/ \mu \mathrm{L}$, as observed by electrophoretic analysis. The accuracy of the LAMP reaction was confirmed by restriction endonuclease digestion as well as by direct sequencing of the amplified product. This method can specifically detect $M$. hyopneumoniae; other species with high homology and other bacterial and virus strains gave negative results. To test the utility of this procedure, the LAMP assay was applied to 40 clinical samples collected from swine lung tissues experimentally challenged with $M$. hyopneumoniae isolates, and compared to the results from a real-time polymerase chain reaction (PCR) assay. A concordance of $100 \%$ was observed between the two assays. In conclusion, the results from our study demonstrated that the LAMP assay provided a rapid reaction and was inexpensive to perform, with no need of complex instruments or systems such as Geneamp PCR. The LAMP assay may therefore be applied in routine diagnosis in the clinical laboratory and for in-field detection of M. hyopneumoniae infection.

Key words: $p 36$ gene; Loop-mediated isothermal amplification; Mycoplasma hyopneumoniae

\section{INTRODUCTION}

A high proportion of farms with respiratory problems among their stock are infected with Mycoplasma hyopneumoniae, which is the primary agent of enzootic pneumonia of swine, a worldwide chronic respiratory disease in pig farms characterized by high morbidity and low mortality (Kobisch and Friis, 1996; Maes et al., 1996). The economic impact of mycoplasma pneumonia of swine is considerable, due mainly to reduced growth and feeding efficiency (Straw, 1990; Paisley et al., 1993), and an increased susceptibility of pigs to infection by other organisms (Kobisch and Friis, 1996). Currently, clinical examination is usually considered to be of low value for diagnosis, in particular when compared to the direct detection of M. hyopneumoniae in lung lesions by polymerase chain reaction (PCR) amplification. In fattening pigs, a quantitative assessment of the onset of coughing (typically dry and nonproductive) improves the diagnosis of enzootic pneumonia and can occasionally substitute for the detection of M. hyopneumoniae by PCR (Nathues et al., 2012). M. hyopneumoniae culture is laborious, time-consuming, and requires several weeks to produce results; frequently, the culture media can become overgrown with M. hyorhinis or M. flocculare as well (DorigoZetsma et al., 1999). Serological methods are insufficiently sensitive and cannot distinguish between vaccinated and $M$. hyopneumoniae-infected pigs in field screening (Moitinho-Silva et al., 2012; Simionatto et al., 2012). More rapid, higher sensitivity methods were therefore developed, one of them being the PCR technique for M. hyopneumoniae DNA detection (Dorigo-Zetsma et al., 1999; Lu et al., 2010; Shen et al., 2011). In the last few years, realtime PCR methods for the diagnosis of M. hyopneumoniae have been described (Dubosson et al., 2004). The advantage of this application, compared to conventional PCR methods, is its higher speed and reduced requirement in handling of PCR products such as needed for 
electrophoretic analysis. However, owing to the expensive systems required, this application is still not very common in hospital-based laboratories.

Notomi et al. (2000) reported a novel nucleic acid amplification method called loopmediated isothermal amplification (LAMP), which is capable of amplifying DNA under isothermal conditions with high specificity, efficiency, and speed. The most significant advantage of LAMP is the ability to amplify specific sequences of DNA under isothermal conditions between $60^{\circ}$ and $65^{\circ} \mathrm{C}$, thereby obviating the need for a thermal cycler (Notomi et al., 2000). Moreover, the LAMP assay synthesizes a large amount of by-product, an insoluble white precipitate of magnesium pyrophosphate, which can be judged visually through the appearance of a white precipitate, or by fluorescence when observed under ultraviolet light following the addition of SYBR Green dye. The LAMP reaction does not require a complex thermocycler; rather, a simple water bath is sufficient for the entire process (Yamazaki et al., 2008; Fu et al., 2011).

The aim of the current study was to develop a LAMP assay specific for M. hyopneumoniae in order to facilitate its rapid, sensitive, and reliable detection and/or identification from clinical samples. Such an assay would be an invaluable tool for both animal health studies and pathogen detection.

\section{MATERIAL AND METHODS}

\section{Bacterial strains and clinical specimens}

For the evaluation of primer specificity, bacteria and virus were prepared from 12 reference strains: M. hyopneumoniae 168 strain, M. hyorhinis, M. flocculare, M. hyosynoviae, Actinobacillus pleuropneumoniae, Streptococcus suis, Haemophilus parasuis, swine Pasteurella multocida, classical swine fever virus (CSFV), porcine circovirus type 2 (PCV2), porcine reproductive and respiratory syndrome virus (PRRSV), and porcine pseudorabies virus (PRV), preserved by the Key Laboratory of Animal Diseases Diagnostic and Immunology of Ministry of Agriculture, Institute of Veterinary Medicine, Jiangsu Academy of Agricultural Sciences.

\section{Reagents and materials}

The TIANamp DNA Kit was a produced by the Tiangen Biotech Co., Ltd. (Beijing, China). Bst DNA polymerase and betaine were purchased from New England Biolabs (Ipswich, MA, USA) and Sigma-Aldrich Chemical Company (Hattiesburg, MS, USA), respectively. MobI restriction endonuclease was a product of TaKaRa BioTechnology Co., Ltd. (Dalian, China). SYBR Green I was obtained from Invitrogen Corporation (Carlsbad, CA, USA).

\section{Design of primers for LAMP}

Three sets of oligonucleotide primers were used for the $M$. hyopneumoniae LAMP assay and were designed based upon the $M$. hyopneumoniae $p 36$ conserved region using the LAMP primer design software Premier 5.0 (http://primerexplorer.jp/elamp4.0.0/index.html; Eiken Chemical Co. Ltd.; Taito-ku, Japan). Each set consisted of four primers: an outer (F3 and B3) and an inner (FIP and BIP) primer pair. Primer sequences are shown in Table 1. 
Table 1. Three sets of primers for detection of Mycoplasma hyopneumoniae in loop-mediated isothermal amplification assays.

\begin{tabular}{ll}
\hline Primers & Sequences $\left(5^{\prime}-3^{\prime}\right)$ \\
\hline $361 \mathrm{~F} 3$ & TGC ATC CGA GTA TGG AAT T \\
$361 \mathrm{~B} 3$ & TTC AAG CCG AGT TTC ACC \\
$361 \mathrm{FIP}$ & GCA AAG AAG CTG AGG CAT CTT CGA TAT TAA TCC TGA TTT TGC CGA T \\
$361 \mathrm{BIP}$ & CCG ATT AGT GTC TCC CGT TAT GAT TTT TGT GGT CTT CCC GC \\
$362 \mathrm{~F} 3$ & AGT ATT ATT GTT GCT AAT CCT GT \\
$362 \mathrm{~B} 3$ & GTT CAC CCA TCA CGT AGG \\
$362 \mathrm{FIP}$ & CAC TAC CGA TAA CTT TTT GAT CGG ATG ATA TAA TTA CAA GGG CTT ACC \\
$362 \mathrm{BIP}$ & GAA CTG TTT TAG ATA CAG CAA GGC TTG AAC CGA ATT AGG CGA TAC \\
$363 \mathrm{~F} 3$ & CTG ATA ACA TCC GAA TTA TCC G \\
$363 \mathrm{~B} 3$ & CCT GAA CCG AAT TAG GCG \\
$363 \mathrm{FIP}$ & CCG GTA AGC CCT TGT AAT TAT ATC ATG CAC TAAAAG TCA AAG AAA GT \\
$363 \mathrm{BIP}$ & CAT CTG GAT TTT CCG ATC AAA AAG TCG ATT GCA AAT TGA AGC CT \\
\hline
\end{tabular}

\section{DNA and cDNA from M. hyopneumoniae and other strains}

The amplified $p 36$ PCR product was purified and cloned into the vector pET-28a $(+)$ for the construction of a $p 36$ expression vector [pET-28a (+)-p36]. Purified plasmid DNA, and genomic DNA and cDNA from $M$. hyopneumoniae and other strains were extracted from bacterial cultures with a commercial DNA/RNA extraction kit (Tiangen), following the manufacturer instructions, for detection of LAMP assay specificity. M. hyopneumoniae genomic DNA from lung tissues was prepared via the boiling method. The resulting supernatant was used as a template for determining the clinical application of the LAMP assay $v s$ real-time PCR.

\section{LAMP reaction}

pET-28a (+)-p36 plasmid DNA was used for the LAMP assay as follows: the reaction mixture consisted of a final volume of $25 \mu \mathrm{L}$ containing $\mathrm{ddH}_{2} \mathrm{O}, 10 \mathrm{X}$ ThermoPol buffer $(2.5$ $\mu \mathrm{L}$ ), $4 \mathrm{mM} \mathrm{MgSO}, 0.2 \mu \mathrm{M}$ of each primer $\mathrm{F} 3$ and $\mathrm{B} 3,0.8 \mu \mathrm{M}$ of each primer FIP and BIP, $0.48 \mathrm{M}$ betaine, $1.2 \mathrm{mM}$ dNTP mixture, $12 \mathrm{U}$ Bst DNA polymerase, and $1 \mu \mathrm{L}$ template DNA. LAMP primers were optimized with 361,362 , and 363 primers by incubating the mixture at $61^{\circ}, 63^{\circ}$, and $65^{\circ} \mathrm{C}$ for $45 \mathrm{~min}$, respectively. The reaction was terminated via heating at $80^{\circ} \mathrm{C}$ for $5 \mathrm{~min}$. A negative control (replacement of the template with $\mathrm{H}_{2} \mathrm{O}$ ) was included in each run. The reaction mixture was visually checked for a white precipitate, followed by electrophoresis of the LAMP products $(5 \mu \mathrm{L})$ in $1.5 \%$ agarose gels containing SYBR Green I to determine the optimal reaction conditions. All experiments were repeated at least twice. The LAMP product was identified by digestion with the restriction endonuclease MobI. The restriction digestion system consisted of $25 \mu \mathrm{L}$ LAMP products, $4 \mu \mathrm{L} 10 \mathrm{X} \mathrm{K}$ Buffer, $4 \mu \mathrm{L} \mathrm{MobI}$, and $7 \mu \mathrm{L} \mathrm{ddH_{2 }} \mathrm{O}$. The length of the digested restriction fragment was $188 \mathrm{bp}$.

\section{Real-time PCR amplification}

A 1- $\mu$ L DNA aliquot from the targeted organism was used for real-time PCR in a 25$\mu \mathrm{L}$ final volume containing $2 \mathrm{X}$ Premix $(12.5 \mu \mathrm{L})$ [TaKaRa Biotechnology (Dalian) Co., Ltd.] $0.05 \mu \mathrm{M}$ probes, $50 \mathrm{X}$ Rox $0.5 \mu \mathrm{L}$, and $0.4 \mu \mathrm{M}$ of each primers $\mathrm{F}$ and $\mathrm{R}$. The reaction included an initial PCR activation step at $95^{\circ} \mathrm{C}$ for 10 min followed by 40 cycles of denaturation at $95^{\circ} \mathrm{C}$ 
for $20 \mathrm{~s}$ and combined annealing/extension at $60^{\circ} \mathrm{C}$ for $1 \mathrm{~min}$. The PCR products were detected with 7500 instrument (Applied Biosystems). Subsequent data analysis was performed using the CFX manager software.

\section{Sensitivity of the LAMP assay}

To determine the sensitivity of the LAMP and real-time PCR assays, serial dilutions of plasmid pET-28a (+)-p36, ranging from $10^{6}$ to $10^{-1}$ copies $/ \mu \mathrm{L}$ were amplified. The LAMP sensitivity test was conducted under the optimal reaction conditions containing $1 \mu \mathrm{L}$ diluted DNA templates. The LAMP reaction was performed at $63^{\circ} \mathrm{C}$ for $45 \mathrm{~min}$. Negative and positive controls were performed using sterile water and genomic DNA template, respectively. For detection of LAMP products, the reaction tubes were observed both visually and under ultraviolet illumination, and the final reaction mixtures $(5 \mu \mathrm{L})$ run on a $1.5 \%$ agarose gel were visualized under ultraviolet light illumination as well following SYBR Green I staining.

\section{Specificity of the LAMP assay}

The specificity of the LAMP assay was first determined with $M$. hyopneumoniae DNA templates. A negative control was included in each run using $\mathrm{ddH}_{2} \mathrm{O}$ as the template. In addition, other species with high homology to M. hyopneumoniae, including M. hyorhinis, M. flocculare, M. hyosynoviae, Actinobacillus pleuropneumoniae, Streptococcus suis, Haemophilus parasuis, swine Pasteurella multocida, CSFV, PCV2, PRRSV, and PRV were tested to further assess the specificity of the LAMP assay. The LAMP reactions were performed at $63^{\circ} \mathrm{C}$ for 45 min. The LAMP products was electrophoresed on $1.5 \%$ agarose gels containing SYBR Green $\mathrm{I}$, and detected as described above.

\section{Application of LAMP assays on clinical samples}

The applicability of the LAMP assay to detect M. hyopneumoniae was assessed by comparison of the detection results between LAMP and real-time PCR using field samples. Pigs were obtained from a source known to be negative for M. hyopneumoniae. All animal procedures were approved by the Animal Ethics Committee of Jiangsu Academy of Agricultural Sciences. The experiments were performed in Jiangsu Academy of Agricultural Sciences. Fifty pigs were employed for the study and divided into five groups. Forty pigs were challenged by delivering $5 \mathrm{~mL} 10^{-2}$ to $10^{-5}$-diluted $M$. hyopneumoniae $\mathrm{NJ}$ strain culture by intratracheal injection. As a control group, 10 pigs were injected with phosphate-buffered saline into the trachea. Pigs were monitored twice daily for a minimum of $15 \mathrm{~min}$ for clinical signs of illness. Clinical signs and lung lesions were observed 25 days post challenge. Eight pigs from each group were randomly slaughtered and the lungs were collected for LAMP and PCR assays. M. hyopneumoniae genomic DNA from pig lung tissues was prepared by boiling for 10 min. After centrifugation, the resulting supernatant was used as DNA templates.

\section{RESULTS}

\section{Optimization of LAMP assay conditions}

The specific amplification generated many ladder-like patterned bands on the agarose 
gel. Using the 361 and 362 primers, amplification was initially detected at $61^{\circ}, 63^{\circ}$, and $65^{\circ} \mathrm{C}$, and showed higher levels of DNA resolution at $63^{\circ} \mathrm{C}$ when compared to other temperatures. However, no amplification could be detected from the 363 primers. Clearer ladder-like patterned bands (Figure 1A) and a white precipitate (Figure 1B) were observed at $63^{\circ} \mathrm{C}$ with the 362 primers when compared to other temperatures and primer sets.

A

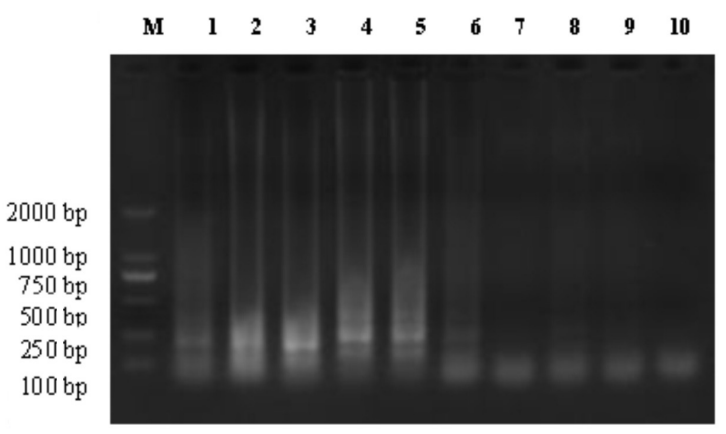

B

N1 N2

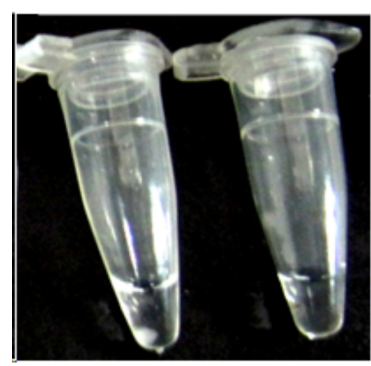

C

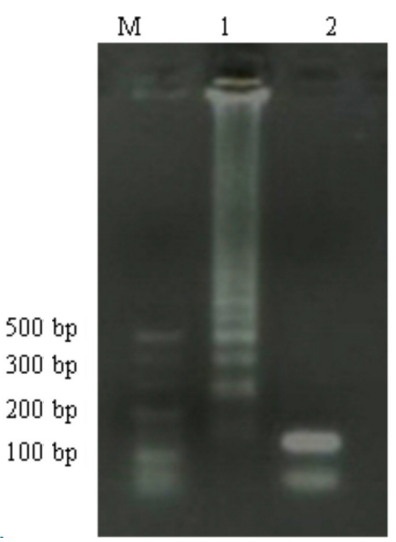

Figure 1. Optimization of the loop-mediated isothermal amplification (LAMP) procedure. A. Electropherogram determination of optimal LAMP primer pairs and reaction temperature. Lane $M=$ DL2000 DNA marker; lanes 1-3=361 primers, at $61^{\circ}, 63^{\circ}$, and $65^{\circ} \mathrm{C}$, respectively; lanes $4-6=362$ primers, at $61^{\circ}, 63^{\circ}$, and $65^{\circ} \mathrm{C}$, respectively; lanes $7-9=363$ primers, at $61^{\circ}, 63^{\circ}$, and $65^{\circ} \mathrm{C}$, respectively; lane $10=$ negative control. B. White precipitate of the optimal LAMP reaction products. $\mathrm{N} 1$ = Mycoplasma hyopneumoniae genomic DNA template; $\mathrm{N} 2$ = negative control. C. Electropherogram of endonuclease digestion identification of the LAMP-amplified product. Lane $M=20$-bp ladder DNA marker; lane $1=$ LAMP product from genomic DNA of M. hyopneumoniae; lane 2 = LAMP product from lane 1 after digestion. 
The accuracy of the LAMP reaction was confirmed by digestion with the restriction enzyme $M o b$ I to ensure that the LAMP products had the sequence corresponding to the $p 36$ gene of M. hyopneumoniae. The product of MobI digestion was $188 \mathrm{bp}$ in size, a finding consistent with the predicted size (Figure 1C).

\section{Sensitivity of the LAMP assays}

The detection limits of the LAMP assay were found to be 10 copies/ $\mu \mathrm{L}$ for a $45 \mathrm{~min}$ reaction at $63^{\circ} \mathrm{C}$ and analysis by agarose gel electrophoresis (Figure $2 \mathrm{~A}$ ) as well as for visualization of a white precipitate (Figure 2B). Real-time PCR demonstrated detection limits of 10 copies (Figure 2C) requiring over $60 \mathrm{~min}$ in the thermocycler. No amplification was apparent in the LAMP reaction when the sample tube lacked target DNA.

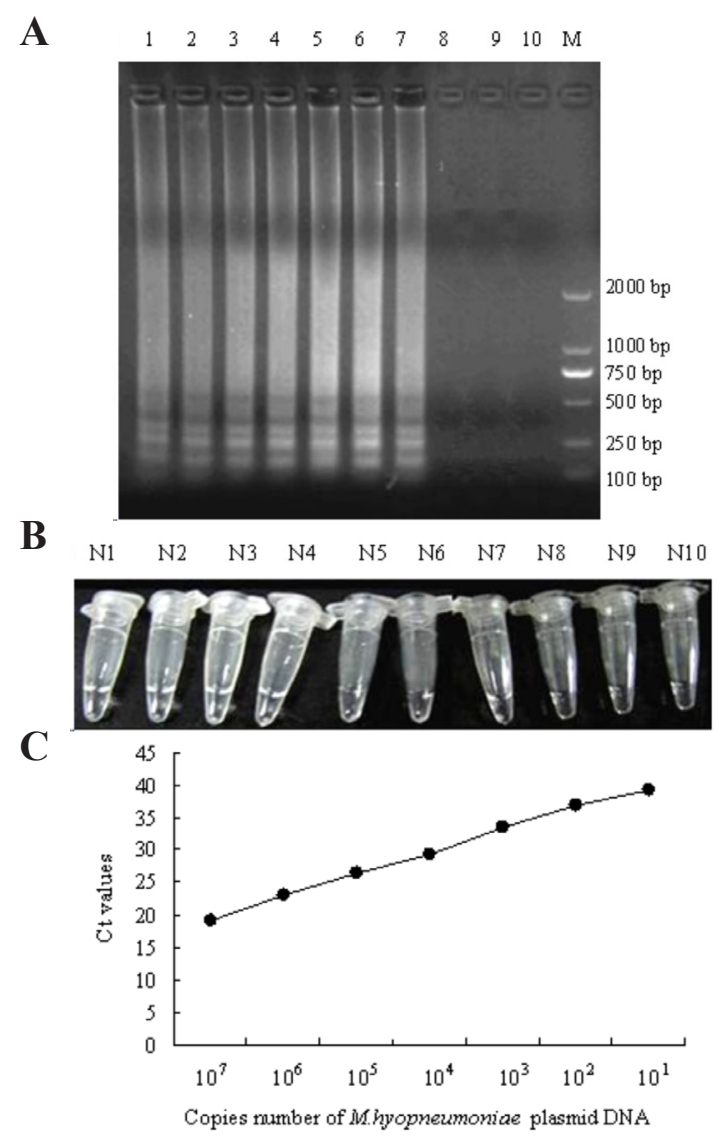

Figure 2. Sensitivity determination of loop-mediated isothermal amplification (LAMP) and real-time polymerase chain reaction (PCR) assays. A. Electropherogram determination of LAMP assay sensitivity. Lane M = DL2000 DNA marker; lane $1=$ Mycoplamsa hyopneumoniae genomic DNA template; lanes $2-9=10^{\mathrm{x}}$ copies $(1 \mu \mathrm{L})$; lane $2=$ $10^{6}$; lane $3=10^{5}$; lane $4=10^{4} ;$ lane $5=10^{3} ;$ lane $6=10^{2} ;$ lane $7=10^{1} ;$ lane $8=10^{0} ;$ lane $9=10^{-1}$; lane $10=$ negative control. B. White precipitates of LAMP amplification products. $\mathrm{N} 1=$ M. hyopneumoniae genomic DNA template; $\mathrm{N} 2-9=10^{\mathrm{x}}$ copies $(1 \mu \mathrm{L}) ; \mathrm{N} 2=10^{6} ; \mathrm{N} 3=10^{5} ; \mathrm{N} 4=10^{4} ; \mathrm{N} 5=10^{3} ; \mathrm{N} 6=10^{2} ; \mathrm{N} 7=10^{1} ; \mathrm{N} 8=10^{0} ; \mathrm{N} 9=10^{-1} ; \mathrm{N} 10$ $=$ negative control. $\mathbf{C}$. Relationship between $\mathrm{Ct}$ values and copy number of $M$. hyopneumoniae plasmid DNA in the real-time PCR assay. Copy numbers were from $10^{7}-10^{1}$ copies $/ \mu \mathrm{L}$. 


\section{Specificity of the LAMP assays}

The LAMP assay specifically amplified DNA from M. hyopneumoniae but not from any of the other Mycoplasma species or other bacterial or viral species. No false-positive amplification was observed, indicating the high specificity of the established LAMP assays in electrophoretic analysis (Figure 3).

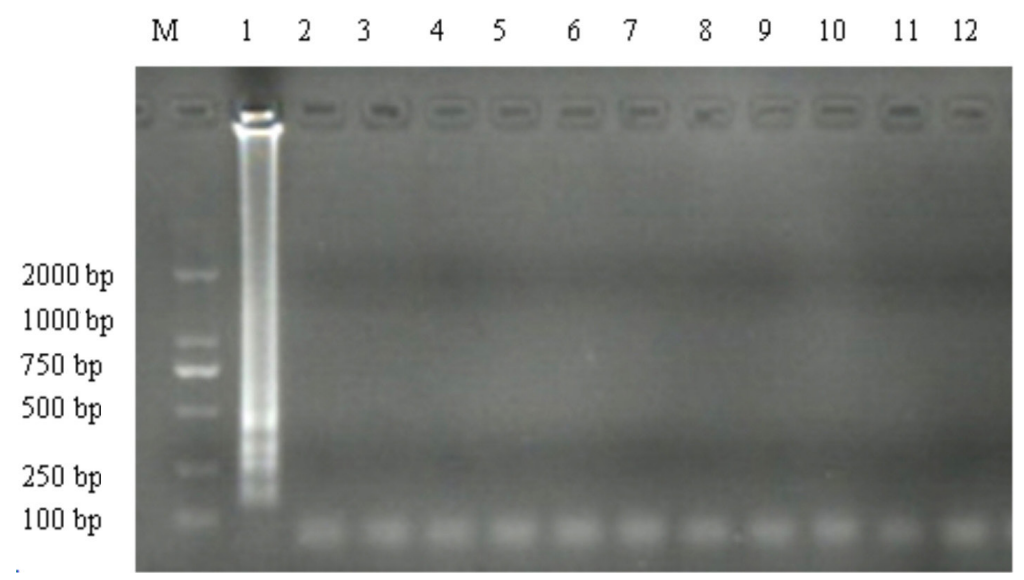

Figure 3. Electropherogram determination of loop-mediated isothermal amplification (LAMP) assay specificity. Lane $M=$ DL2000 DNA marker; lane 1 = Mycoplasma hyopneumoniae genomic DNA template; lane 2 = negative control; lane 3 = Haemophilus parasuis DNA template; lane 4 = Actinobacillus pleuropneumoniae DNA template; lane 5 = Streptococcus suis DNA template; lane $6=$ swine Pasteurella multocida DNA template; lane $7=$ Mycoplasma hyosynoviae DNA template; lane $8=$ Mycoplasma hyorhinis DNA template; lane $9=P R R S V$ cDNA template; lane $10=$ PCV2 DNA template; lane $11=C S F V$ cDNA template; lane $12=P R V$ DNA template. PRRSV $=$ porcine reproductive and respiratory syndrome virus; $\mathrm{PCV} 2=$ porcine circovirus type $2 ; \mathrm{CSFV}=$ classical swine fever virus; PRV = porcine pseudorabies virus.

\section{Application of LAMP assays on clinical samples}

For both LAMP and real-time PCR assays, 35 clinical samples tested positive for $M$. hyopneumoniae. Thus, the concordance between the two assays was $100 \%$. The positive predictive value was determined to be $100 \%$ for both LAMP and real-time PCR assays, with no false positives observed (Table 2). In comparison with real-time PCR methods, LAMP showed a more rapid reaction and a lower cost.

Table 2. Loop-mediated isothermal amplification assay results from Mycoplasma hyopneumoniae clinical samples.

\begin{tabular}{lll}
\hline Samples (total number) & LAMP detection rate $(\%) / \mathrm{N}$ positive samples & Real-time polymerase chain reaction \\
\hline Swine lung tissues $(\mathrm{N}=40)$ & $100 / 35$ & $100 / 35$ \\
\hline
\end{tabular}

\section{DISCUSSION}

Although major efforts have been made to control M. hyopneumoniae infection and its detrimental effects, significant economic losses remain in pig production worldwide due 
to enzootic pneumonia (Thacker, 2006). Effective control requires a number of actions, including establishing and monitoring the extent of disease, reducing transmission of infection, and developing an appropriate vaccination strategy. Establishing and monitoring the extent of enzootic pneumonia in pig herds now typically incorporate enzyme-linked immunosorbent assay, PCR, and slaughter surveillance methods. These technologies, however, have limitations. Furthermore, these molecular diagnostic techniques require either a precise instrument for amplification or an elaborate method for detection of the amplified products. In the present study, we evaluated the LAMP assay for detection of M. hyopneumoniae in either culture isolates or lung tissues. This assay is a simple diagnostic tool in which the reaction takes place in a single tube. In evaluating detection methodologies for epidemiological purposes, a series of attributes should be considered and assessed, including specificity, sensitivity, simplicity, expense, and time. LAMP has been known as a rapid, specific, sensitive, cost-effective, and easy-to perform alternative for diagnosis of pancreatic necrosis virus and porcine circovirus type 1 (Suebsing et al., 2011; Wang et al., 2011), and has been applied to the direct detection of the Mycobacterium tuberculosis complex, Mycobacterium avium and intracellular Mycobacterium, and M. pneumoniae (Iwamoto et al., 2003), and of the severe acute respiratory syndrome coronavirus (Hong et al., 2004).

The genome of $M$. hyopneumoniae encodes several immunodominant proteins, including a cytosolic protein (P36), three membranous proteins (P46, P65, and P74), and an adhesion protein (P97). Cross-reactions with $M$. flocculare and M. hyorhinis reduce the specificity of conventional serological detection methods. However, certain antigenic determinants of the P36 protein have been shown to be specific for M. hyopneumoniae (Caron et al., 2000; Cheikh Saad et al., 2003). The extreme sensitivity provided by P36 may therefore be of substantial diagnostic value in swine mycoplasma pneumonia. To capitalize on this potential, it is very important to find a conserved nucleic acid fragment for designing specific LAMP primers and develop an efficient, accurate LAMP assay (Notomi et al., 2000; Fu et al., 2011). In the present study, two sets of $M$. hyopneumoniae-specific primers were designed with inner and outer primer pairs recognizing six distinct regions, and which amplified only $M$. hyopneumoniae DNA. High specificity was illustrated through lack of false-positive observations for reference strains and $100 \%$ concordance during application. Due to its powerful amplification efficiency, LAMP was characterized by high sensitivity and low detection limits at 10 copies for a 45 -min reaction at $63^{\circ} \mathrm{C}$, and could be performed in a laboratory with limited technology. In comparison, although the real-time PCR detection limit was also 10 copies, this technique commonly requires a costly instrument and nearly $2 \mathrm{~h}$ for amplification reactions alone.

As the reaction was performed under isothermal conditions without a thermal cycler, only simple equipment like a heat block or water baths were needed for the performance of LAMP assays at low expense. Detection time was no more than $1.2 \mathrm{~h}$ for one sample. Therefore, both simplicity and sensitivity make the LAMP assay a simple, rapid, and cost-effective alternative to the current rapid detection methodologies utilized in epidemiologic studies.

In conclusion, this assay was highly specific and showed a sensitivity of 10 copies of M. hyopneumoniae in a 45-min reaction, as determined by visualization of a white precipitation as well as by electrophoretic analysis. Together with its ease of operation, rapid reaction, and inexpensive system, the LAMP assay is therefore more appropriate than the real-time PCR assay for clinical detection of diseases, especially in field conditions and at quarantine inspection stations. 


\section{ACKNOWLEDGMENTS}

Research supported by the Jiangsu Agriculture Science and Technology Innovation Fund (\#CX(13)3066), the National Nature Science Foundation of China (\#31100136), and the Jiangsu Province Postdoctoral Fund (\#6511201).

\section{REFERENCES}

Caron J, Ouardani M and Dea S (2000). Diagnosis and differentiation of Mycoplasma hyopneumoniae and Mycoplasma hyorhinis infections in pigs by PCR amplification of the p36 and p46 genes. J. Clin. Microbiol. 38: 1390-1396.

Cheikh Saad BK, Shareck F and Dea S (2003). Monoclonal antibodies to Escherichia coli-expressed P46 and P65 membranous proteins for specific immunodetection of Mycoplasma hyopneumoniae in lungs of infected pigs. Clin. Diagn. Lab. Immunol. 10: 459-468.

Dorigo-Zetsma JW, Zaat SA, Wertheim-van Dillen PM, Spanjaard L, et al. (1999). Comparison of PCR, culture, and serological tests for diagnosis of Mycoplasma pneumoniae respiratory tract infection in children. J. Clin. Microbiol. 37: 14-17.

Dubosson CR, Conzelmann C, Miserez R, Boerlin P, et al. (2004). Development of two real-time PCR assays for the detection of Mycoplasma hyopneumoniae in clinical samples. Vet. Microbiol. 102: 55-65.

Fu S, Qu G, Guo S, Ma L, et al. (2011). Applications of loop-mediated isothermal DNA amplification. Appl. Biochem. Biotechnol. 163: 845-850.

Hong TC, Mai QL, Cuong DV, Parida M, et al. (2004). Development and evaluation of a novel loop-mediated isothermal amplification method for rapid detection of severe acute respiratory syndrome coronavirus. J. Clin. Microbiol. 42: 1956-1961.

Iwamoto T, Sonobe T and Hayashi K (2003). Loop-mediated isothermal amplification for direct detection of Mycobacterium tuberculosis complex, M. avium, and M. intracellulare in sputum samples. J. Clin. Microbiol. 41: 2616-2622.

Kobisch M and Friis NF (1996). Swine mycoplasmoses. Rev. Sci. Tech. 15: 1569-1605.

Lu XM, Feng ZX, Liu MJ and Wu XS (2010). Establishment of a nested PCR assay for detection of Mycoplasma hyopneumoniae. Jiangsu J. Agric. Sci. 26: 91-95.

Maes D, Verdonck M, Deluyker H and de KA (1996). Enzootic pneumonia in pigs. Vet. Q 18: 104-109.

Moitinho-Silva L, Heineck BL, Reolon LA, Paes JA, et al. (2012). Mycoplasma hyopneumoniae type I signal peptidase: expression and evaluation of its diagnostic potential. Vet. Microbiol. 154: 282-291.

Nathues H, Spergser J, Rosengarten R, Kreienbrock L, et al. (2012). Value of the clinical examination in diagnosing enzootic pneumonia in fattening pigs. Vet. J. 193: 443-447.

Notomi T, Okayama H, Masubuchi H, Yonekawa T, et al. (2000). Loop-mediated isothermal amplification of DNA. Nucleic Acids Res. 28: E63.

Paisley LG, Vraa-Andersen L, Dybkjaer L, Moller K, et al. (1993). An epidemiologic and economic study of respiratory diseases in two conventional Danish swine herds. II: Associations between lesions present at slaughter and mean daily gains during specific intervals of the growth period. Acta Vet. Scand. 34: 331-344.

Shen LJ, Li W and He YH (2011). Molecular diagnosis of toxoplasmosis. Zhongguo Xue Xi Chong Bing Fang Zhi Za Zhi 23: 465-469.

Simionatto S, Marchioro SB, Galli V, Brum CB, et al. (2012). Immunological characterization of Mycoplasma hyopneumoniae recombinant proteins. Comp. Immunol. Microbiol. Infect. Dis. 35: 209-216.

Straw BE (1990). Effect of Campylobacter spp-induced enteritis on growth rate and feed efficiency in pigs. J. Am. Vet. Med. Assoc. 197: 355-357.

Suebsing R, Oh MJ and Kim JH (2011). Evaluation of rapid and sensitive reverse transcription loop-mediated isothermal amplification method for detecting infectious pancreatic necrosis virus in chum salmon (Oncorhynchus keta). J. Vet. Diagn. Invest. 23: 704-709.

Thacker EL (2006). Lung inflammatory responses. Vet. Res. 37: 469-486.

Wang C, Pang VF, Jeng CR, Lee F, et al. (2011). Detection of porcine circovirus type 1 in commercial porcine vaccines by loop-mediated isothermal amplification. Folia Microbiol. 56: 483-489.

Yamazaki W, Seto K, Taguchi M, Ishibashi M, et al. (2008). Sensitive and rapid detection of cholera toxin-producing Vibrio cholerae using a loop-mediated isothermal amplification. BMC Microbiol. 8: 94. 